2 Hodson ME, Penketh ARL, Batten JC. Aerosol carbenicillin and gentamicin treatment of Pseudomonas aeruginosa infection in patients with cystic fibrosis. Lancet 1981 ; ii : 1137-9.

3 Reas HW. The effect of N-acetylcysteine on the viscosity of tracheobronchial secretions in cystic fibrosis of the pancreas. J Pediatr 1963;62:31-5.

4 Lawson D, Saggers BA. NAC and antibiotics in cystic fibrosis (letter). Br Med J 1965;i:317.

5 Roberts DE, Cole $P$. The carbenicillin potentiating effect of N-acetylcysteine in vitro. Eur J Pediatr 1981;137:78.
6 Hancock REW, Carey AM. Outer membrane of Pseudomonas aeruginosa: heat- and 2-mercaptoethanol-modifiable proteins. J Bacteriol 1979;140:902-10.

Correspondence to Dr D P Heaf, Respiratory Laboratory, The Hospital for Sick Children, Great Ormond Street, London WC1N 3JH.

Received 13 June 1983

\title{
Endotoxaemia in cystic fibrosis: response to antibiotics
}

\author{
G TAYLOR, C STERN, M SILVERMAN, AND M MEARNS
}

Department of Paediatrics and Neonatal Medicine and Department of Immunology, Royal Postgraduate Medical School, Hammersmith Hospital, and Queen Elizabeth Hospital for Children, London

SUMMARY In a study of 6 children with cystic fibrosis receiving intravenous antibiotics for pseudomonas lung infection, serum endotoxin values were monitored by a modification of the limulus lysate technique. The values fell with treatment, reflecting a response that was not always apparent on clinical assessment. Endotoxin concentrations may offer a more precise way of monitoring the effects of antibiotic treatment in CF patients.

As the respiratory tract of children with cystic fibrosis (CF) becomes colonised with Pseudomonas aeruginosa there is usually an associated clinical deterioration. It is because of this association that attempts are often made to control Ps. aeruginosa infection by courses of intravenous antibiotics, although it is questionable whether their effect is valuable or prolonged. ${ }^{1}$ It is difficult, using routine bacteriological methods, to assess the true extent of the infection since quantitative sputum bacteriology is imprecise. Moreover, the extent of lung damage produced by $P$ s. aeruginosa and the effect of antibacterial treatment can only be assessed indirectly.

All Gram negative organisms produce endotoxin, a part of their cell wall. It is a high molecular weight complex of lipid, polysaccharide, and protein ${ }^{2}$ and the bioactive part of the molecule is remarkably conserved among most Gram negative organisms. This means that the biological effects of endotoxin from most of these organisms are very similar despite species differences.

We have developed a rapid, sensitive assay for endotoxin which depends upon its biological activity, and wished to see whether endotoxin was detectable in the serum of patients with exacerbation of CF, and if so, whether the value changed in response to treatment.

\section{Patients and methods}

As part of a wider study of bacterial antigenaemia in CF, 6 children with CF (four girls and two boys) with ages ranging from 6-19 years were studied. They were admitted to hospital for antibiotic treatment either for an acute exacerbation or in an attempt to improve respiratory function before a new school term. They received their usual diet and physiotherapy. Antibiotic treatment consisted of gentamycin ( $3 \mathrm{mg} / \mathrm{kg} / 8$ hourly, IV) and carbenicillin ( $50 \mathrm{mg} / \mathrm{kg} / 8$ hourly, IV) for $10-14$ days.

A clinical scoring system, grading symptoms $0-3$ for cough, shortness of breath, and sputum production was devised. Peak expiratory flow rate was measured daily and the growth of Ps. aeruginosa from sputum was classed as absent, light, moderate, or heavy.

Blood for endotoxin assay was taken with routine specimens before treatment, after 5-7 days treatment, and where possible after completion of the antibiotic course. Specimens were collected by clean venepuncture into endotoxin free glass bijou bottles, allowed to clot at room temperature, and separated within one hour. Serum was then stored at $-20^{\circ} \mathrm{C}$ until assayed.

The assay ${ }^{3}$ was a modification of the limulus assay, using the chromogenic substrate $\mathbf{S 2 2 2 2}$ (Kabi Diagnostics). The chromogenic substrate increases the sensitivity of the limulus assay and enables quantitative results to be obtained. ${ }^{4}$ Before assay the samples were diluted $1: 4$ with pyrogen free water and heated to $68^{\circ} \mathrm{C}$ for 30 minutes to destroy any enzyme activity. Ten $\mu \mathrm{l}$ samples and dilutions of the WHO 
Shigella dysenteriae reference standard endotoxin, cach in triplicate, were placed in microtitre wells with $50 \mu \mathrm{l}$ limulus lysate (Pyrostat-Millipore) and incubated at $37^{\circ} \mathrm{C}$ for one hour. Fifty $\mu l$ of $1 \mathrm{mmol}$ dilution of the substrate was then added and the colour change measured using the Multiskan multichannel photometer. Results were calculated from a standard curve, constructed with the reference standard controls and expressed as a quantitative endotoxic activity (relative to the shigella).

\section{Results}

Although there was an overall clinical improvement after treatment, the clinical scores did not show a consistent improvement (Table). Similarly, the peak expiratory flow rate changed little. The amount of Ps. aeruginosa isolated from sputum fell a little, but in no case disappeared.

Endotoxin activity was detectable in all patients before treatment and fell below limits of the assay

Table Clinical scores before, during, and after treatment (mean values and ranges)

\begin{tabular}{llll}
\hline & \multicolumn{1}{l}{ Timing of clinical assessments } \\
\cline { 2 - 4 } & $\begin{array}{l}\text { Before } \\
\text { treatment }\end{array}$ & $5-7$ days & $10-14$ days \\
\hline Cough & $2 \cdot 5(2-3)$ & $1 \cdot 5(1-2)$ & $1 \cdot 5(1-2)$ \\
Sputum & $2 \cdot 6(2-3)$ & $2 \cdot 2(2-3)$ & $2 \cdot 0$ \\
Pseudomonas in sputum & $2 \cdot 6(2-3)$ & $2 \cdot 3(1-3)$ & $2 \cdot 3(1-3)$ \\
\hline
\end{tabular}

Range of scores $0-3$ for each variable.

For number of observations, see Figure.

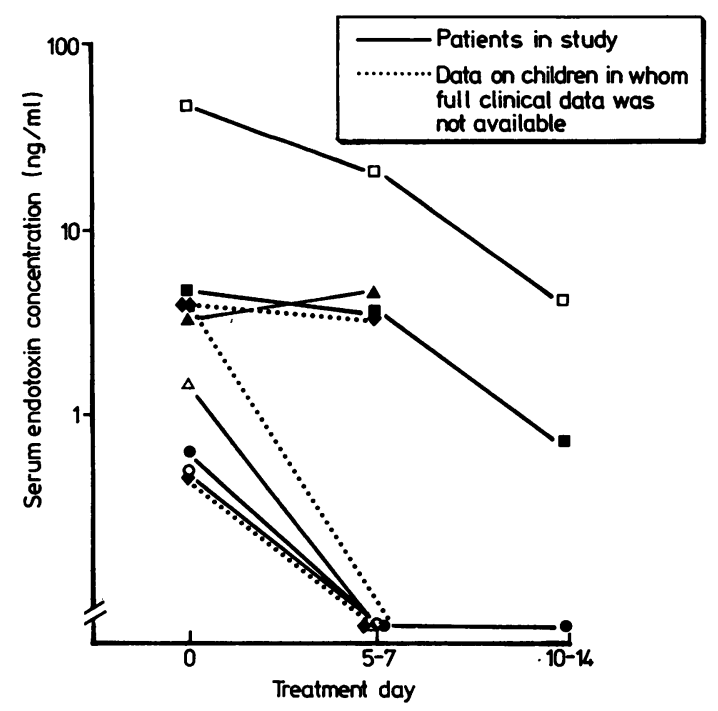

Figure Serum endotoxin values before and after antibiotic treatment in 9 children with cystic fibrosis. after five days in 3 of 6 patients (Figure). In another patient, who had the highest value of the 6 , the concentration fell sieadily with treatment but remained measurable even after treatment finished. This child was one of those admitted for a preschool boost. In two of the children, endotoxin values did not change over the first 5-7 days of treatment, although a subsequent value was lower in one of these children for whom further data was available.

\section{Discussion}

While most studies have tried to relate endotoxaemia to bacteraemia, in CF patients pseudomonas bacteraemia would be unusual. An active infection with a large pool of Gram negative organisms in the respiratory tract is likely to give rise to endotoxaemia, although not necessarily at a symptomatic level. Our method, which is more sensitive than the standard limulus assay, was able to pick up values of endotoxin below those at which frank clinical symptoms were likely ${ }^{5}$ in all of the patients studied. It is not surprising that endotoxin values correlated poorly with the growth of pseudomonas from the sputum, as our assessment of the degree of colonisation was simplistic. Quantitative bacterial counts of sputum are also difficult to interpret reliably and may not be totally representative of the lower respiratory tract flora.

We are not aware of any previous reports of endotoxin in CF. Our results suggest that endotoxaemia reflects appreciable infection rather than subclinical colonisation, as detection of endotoxin implies absorption, presumably across the locally damaged pulmonary epithelium, and the fall in values during antibiotic treatment implies a response to treatment.

It is unlikely that the endotoxin detected arose from the gut, as such endotoxin that is absorbed is normally adequately cleared by the liver ${ }^{6}$ and systemic endotoxaemia is found only with pronounced liver disease. None of our patients had any clinical or biochemical signs of liver dysfunction; nor did they have other signs of infection from other sites as a possible cause for the detected endotoxin.

We suggest that detection of endotoxin may be useful in monitoring antipseudomonas antibiotic treatment in patients with CF, in determining which patients to treat, and in deciding the optimal length of treatment.

G T was supported by a grant from the Cystic Fibrosis Trust Kabi Diagnostics supplied the substrates.

\section{References}

1 Beaudry PH, Marks MI, McDougall D, Desmond K. Rangel $R$. Is anti-pseudomonas therapy warranted in 
acute respiratory exacerbations in children with cystic fibrosis? J Pediatr 1980;97:144-7.

2 Westphal O. Bacterial endotoxins. Int Arch Allergy Appl Immunol 1975;49:1-43.

3 Taylor G, Slade M, Stern C, Silverman M. A new micro-method of endotoxin detection. J Appl Bacteriol $1981 ; 51$ :XX.

4 Webster CJ. Principles of a quantitative assay for bacterial endotoxins in blood that uses limulus lysate and a chromogenic substrate. J Clin Microbiol 1980;12:644-50.
${ }^{5}$ C 4 ridis D, Reinhold RB, Woodruff PWH, Fine J. Endotoxaemia in man. Lancet $1972 ; \mathrm{i}: 1381-6$.

6 Prytz H, Holtst-Christensen J, Korner B, Liehr H. Portal venous and systemic endotoxaemia in patients withou liver disease and systemic endotoxaemia in patients with cirrhosis. Scand J Gastroenterol 1976;11 :857-63.

Correspondence to Dr M Silverman, Royal Postgraduate School of Medicine, Hammersmith Hospital, Ducane Road, London W12 0HS.

Received 11 July 1983

\title{
Three successfully treated cases of nonamoebic liver abscess
}

\author{
P K H TAM, H SAING, AND J T K LAU \\ Division of Paediatric Surgery, Department of Surgery, University of Hong Kong
}

SUMMARY Three previously healthy children developed nonamoebic liver abscess. Diagnosis was aided by liver scan, ultrasound assessment, and computed tomography. A variety of surgical procedures were successful in achieving proper drainage, resection of chronic lesions, and resolution of associated cholangitis.

Nonamoebic liver abscess in children is an uncommon occurrence and only about 100 cases have so far been reported. ${ }^{1-4}$ Most of these cases occurred in compromised hosts with either underlying malignancy, septicaemia, or chronic granulomatous disease and only about $20 \%$ in previously healthy children. ${ }^{1}$ Mortality is high-a figure of $34 \%$ was reported by Cushid ${ }^{1}$ in his review of the published reports. In the past 10 years we have treated three uncompromised children with nonamoebic liver abscesses with satisfactory outcome; each patient requiring a different surgical approach. The need for awareness of this condition and individual management is stressed.

\section{Case reports}

Case 1. An 18 month old boy presented with fever of $1 \frac{1}{2}$ months duration. He had no abdominal pain; there was right upper quadrant tenderness but the liver was not palpable. Blood and liver function tests were all normal, but his chest radiograph showed elevation of the right hemidiaphragm and a liver scan showed a lesion in the right lobe.

At laparotomy an abscess was confirmed; the rest of the liver being studded with small chronic septic nodules. The common bile duct was dilated and packed with 14 ascaris and purulent bile. These were removed and the common duct and abscess drained separately. Escherichia coli was found in both pus and bile. He made an uneventful recovery after a course of antibiotics and antihelminths, and at follow up 8 years later his cholangiogram and liver scan were normal.

Case 2. A 9 year old boy was referred after 6 months of swinging fever, chills, and rigors. He had had two mild attacks of abdominal pain not requiring treatment. Examination showed a tender enlarged liver. Initial investigations in another hospital showed a mild anaemia, raised erythrocyte sedimentation rate $(85 \mathrm{~mm}$ in the first hour), alkaline phosphatase $(358 \mu \mathrm{mol} / 1(50 \mathrm{KA} \mathrm{U} / 100 \mathrm{ml}))$, serum aspartate transaminase $(100 \mu \mathrm{mol} / \mathrm{l}(208 \mathrm{Rf} \mathrm{U} / \mathrm{ml}))$, serum alanine transaminase $(80 \mu \mathrm{mol} / 1$ (167 $\mathrm{Rf}$ $\mathrm{U} / \mathrm{ml})$ ). Leucocyte count, bilirubin, albumin, blood culture, and an ultrasound scan of the liver were all normal. After prolonged empirical antibiotic treatment failed he was referred to this hospital where we repeated ultrasound and found a $4 \mathrm{~cm}$ lesion in the right lobe. A liver scan showed similar results.

At laparotomy there were multiple old abscesses throughout the liver, with three considerably larger lesions-two on the right and one on the leftmeasuring $6 \mathrm{~cm}, 5 \mathrm{~cm}$, and $3 \mathrm{~cm}$ at their largest diameter. All were necrotic granulomata and mainly solid. Wedge excisions of the larger granulomata were performed. Cultures were all sterile. The boy developed a subphrenic haematoma requiring evacuation and a right pleural effusion but recovered well after a three week course of antibiotics. Two and a half years later he was well and had a normal liver scan.

Case 3. A 12 year old girl complained of fever, chills, and rigors for one month but had no abdominal pain. Examination showed a palpable liver edge and right upper quadrant tenderness. She had leucocytosis $\left(16 \times 10^{9} / 1\right) ; 16000 / \mathrm{mm}^{3}$ and a raised alkaline phosphatase $(361 \mu \mathrm{mol} / 1$ (51 KA U/100 ml)) but other parameters of liver function were all normal. Liver scan showed a solitary $5 \mathrm{~cm}$ lesion in right lobe. Computed tomography showed multiple low 